\title{
Optimized sequencing depth and de novo assembler for deeply reconstructing the transcriptome of the tea plant, an economically important plant species
}

\author{
Fang-Dong $\mathrm{Li}^{1,2+}$, Wei Tong ${ }^{1 \dagger}$, En-Hua Xia ${ }^{1 *}$ and Chao-Ling Wei ${ }^{1 *}$ (D)
}

\begin{abstract}
Background: Tea is the oldest and among the world's most popular non-alcoholic beverages, which has important economic, health and cultural values. Tea is commonly produced from the leaves of tea plants (Camellia sinensis), which belong to the genus Camellia of family Theaceae. In the last decade, many studies have generated the transcriptomes of tea plants at different developmental stages or under abiotic and/or biotic stresses to investigate the genetic basis of secondary metabolites that determine tea quality. However, these results exhibited large differences, particularly in the total number of reconstructed transcripts and the quality of the assembled transcriptomes. These differences largely result from limited knowledge regarding the optimized sequencing depth and assembler for transcriptome assembly of structurally complex plant species genomes.
\end{abstract}

Results: We employed different amounts of RNA-sequencing data, ranging from 4 to 84 Gb, to assemble the tea plant transcriptome using five well-known and representative transcript assemblers. Although the total number of assembled transcripts increased with increasing sequencing data, the proportion of unassembled transcripts became saturated as revealed by plant BUSCO datasets. Among the five representative assemblers, the Bridger package shows the best performance in both assembly completeness and accuracy as evaluated by the BUSCO datasets and genome alignment. In addition, we showed that Bridger and BinPacker harbored the shortest runtimes followed by SOAPdenovo and Trans-ABySS

Conclusions: The present study compares the performance of five representative transcript assemblers and investigates the key factors that affect the assembly quality of the transcriptome of the tea plants. This study will be of significance in helping the tea research community obtain better sequencing and assembly of tea plant transcriptomes under conditions of interest and may thus help to answer major biological questions currently facing the tea industry.

Keywords: Tea plant, Camellia sinensis, Transcriptome, de novo assembly, Sequencing depth

\section{Background}

The power of RNA sequencing (RNA-seq) is the fact that the twin aspects of discovery and quantification can be combined in a single high-throughput RNA sequencing experiment. With the rapid innovation of sequencing technology, the scale of raw data generation is

\footnotetext{
* Correspondence: weichl@ahau.edu.cn; xiaenhua@gmail.com

${ }^{+}$Fang-Dong Li and Wei Tong contributed equally to this work.

'State Key Laboratory of Tea Plant Biology and Utilization, Anhui Agricultural

University, Hefei 230036, China

Full list of author information is available at the end of the article
}

growing explosively [1]. Increasing the number of reads should increase the level of uniquely mapped reads to facilitate the de novo assembly and quantification analysis, but this approach can also increase the cost of library preparation and sequencing [2], which can be wasteful in terms of data storage and processing. Thus, the determination of a proper sequencing depth is important across all experiments, including RNA-seq, genome resequencing and de novo sequencing. In RNA-seq, transcript identification is accepted as playing an important

(c) The Author(s). 2019 Open Access This article is distributed under the terms of the Creative Commons Attribution 4.0 International License (http://creativecommons.org/licenses/by/4.0/), which permits unrestricted use, distribution, and reproduction in any medium, provided you give appropriate credit to the original author(s) and the source, provide a link to the Creative Commons license, and indicate if changes were made. The Creative Commons Public Domain Dedication waiver (http://creativecommons.org/publicdomain/zero/1.0/) applies to the data made available in this article, unless otherwise stated. 
role in gene discovery, as it is a key intermediate bridge between the genome and proteome. The assembly and annotation of transcripts, especially novel transcripts or alternatively spliced transcripts, is of great importance in RNA-seq analysis [3].

For an organism that lacks a high-quality reference genome, the de novo assembly of its transcriptome is a useful method for researchers to find the transcripts or genes responsive to various treatments and to better understand the expression patterns of the candidate genes. However, two major problems in de novo assembly have obstructed progress in high-throughput transcript identification: 1) most of the transcripts assembled are not full-length, and 2) only a certain proportion of the genes can be assembled. Some genes only show a high expression abundance under specific conditions or treatments, which means that multiple organs or timeseries sampling need to be performed to obtain more transcripts. In addition, the de novo assembled transcripts using the default parameters in most current assemblers are usually short in length, which hampers further sequence-based analysis and experiments. Therefore, a well-assembled, accurate, and comprehensive transcriptome is a prerequisite for the subsequent analysis. The accurate assembly of a transcript is largely determined by the sequencing depth, which leads to the question of how much data should be generated in an RNA-seq experiment to obtain robust results. Some recent reports suggest that approximately 700 million reads are required to obtain accurate quantification of $>$ 95\% of expressed transcripts in a mammalian genome [4], but there are no systematic analyses illustrating the effect of sequencing depth on transcript assembly [5].

Currently, many programs or tools [6-10] have been developed for the assembly of transcriptomic data. Trinity has been widely adopted in many experiments due to its considerably improved assembly performance with an exhaustive enumeration algorithm to search for isoformrepresenting paths in a de Bruijn graph; this type of analysis makes the algorithm highly sensitive to splicing isoforms but suffers from high false positives. Bridger aims to build a bridge between the key ideas of two popular assemblers, the reference-based assembler Cufflinks [11] and the de novo assembler Trinity [6], which specifically generalized the main techniques employed by Cufflinks to overcome the limitations of Trinity. BinPacker assembles full-length transcripts by remodeling the problem of tracking a set of trajectories of items over a splicing graph, which is constructed by employing the techniques used in Bridger with several updates [10].

Tea is the oldest and among the most popular nonalcoholic beverage in the world [12,13]. Tea is commonly produced from the young leaves of tea plants, which are widely grown in more than 52 countries. Currently, tea has increasingly become the major source of income in tea-producing countries [14]. In the last two years, two draft genomes of tea plants $(C$. sinensis var. sinensis and C. sinensis var. assamica) have been released $[15,16]$. Both genomes possessed a giant nuclear genome size of $\sim 3 \mathrm{~Gb}$. With the rapid development of RNA-seq, many transcriptomic data have been generated in tea plants across different developmental stages, various biotic and abiotic stresses [14, 17-30]. However, due to the absence of a previously constructed high-quality reference genome and the complexities of the tea plant genome, it is difficult to de novo assemble a good transcript sets or to identify novel transcripts, even with reference-based analyses. In tea plants, some transcriptomic studies only focused on only one or few tissues [18, 31-40], whereas others did multiple samples [41-49] or even with pooled samples $[17,37,50]$, making it difficult to assemble transcripts in standard. The amount of data generated among different studies also varies from $1 \mathrm{~Gb}$ to 85 $\mathrm{Gb}$ and most of the studies only use a single tool (mostly Trinity) for transcript assembly (Additional file 1: Table S1).

This abundance of transcriptomic studies encourages us to perform a performance evaluation of different assemblers in tea plants. We also assessed the effect of different sequencing depths on the transcriptome assembly of tea plants and attempted to find an optimized data size that performs better in a range extending from $4 \mathrm{~Gb} / 1 \mathrm{~Gb}$ to $84 \mathrm{~Gb} / 11 \mathrm{~Gb}$ for pooled/single samples. We neither aim to provide an exhaustive compilation of resources or software tools nor to indicate the best analysis pipeline, but rather to provide an annotated guideline for RNA-seq data analysis in tea plants and other similar plant species.

\section{Results}

RNA sequencing and de novo assembly using five representative assemblers

To investigate the suitable de novo assembler and preferred sequencing depth for tea plant transcriptome assembly, we previously sequenced the transcriptome of tea plants derived from eight characteristic tissues (apical bud, first young leaf, second young leaf, mature leaf in summer, stem, flower, fruit, and root) using an Illumina Hiseq2000 platform [51]. This approach yielded a total of $94.1 \mathrm{~Gb}$ of raw RNA-seq data with at least 131 million reads per sample. The average GC content was 46.10\% (Additional file 1: Table S2).

We assembled the RNA-seq data using five popular and typical de novo assemblers, including Trinity, Trans-ABySS, SOAPdenovo, BinPacker and Bridger. The parameters used for the analyses were default with the exception of $k$-mer values, which ranged from 19 to 32 . All assemblies were run on a single-node machine with 
1024 GB memory and 8 Intel 12-core processors. The results show that, among the five assemblers, BinPacker harvested the largest number of transcripts $(423,768)$ followed by Trans-ABySS and Trinity assembly, which was approximately 1.8-fold greater than the SOAPdenovo assembly (Table 1 ). The assembled transcripts also varied in length. Bridger assembly exhibited the largest maximum, average and N50 lengths; these were similar in length to those of BinPacker assembly but were considerably larger than those of the Trans-ABySS and Trinity assemblies. The overall length distribution shows that, compared to Trans-ABySS and Trinity assembly, BinPacker and Bridger assemblies harbored the smallest proportions of transcripts shorter than $500 \mathrm{bp}$, and over $25 \%$ of the assembled transcripts were longer than $1 \mathrm{~kb}$ (Fig. 1a). We further investigated the shared and specific transcripts assembled from five assemblers. The results show that all five assemblers host a core set of 61,836 transcripts, and only an average proportion of $23.4 \%$ is assembler specific. (Additional file 1: Figure S1).

We adopt the BUSCO (Benchmarking Universal Single-Copy Orthologs) datasets from plant lineage to assess the completeness of transcriptome assembly. Of the 1440 expected plant BUSCO orthologs, the Bridger assembly identified 92.5 and $3.8 \%$ as complete and fragmented, respectively; only $3.7 \%$ were considered missing (Fig. 1b). These results were comparable to those of the BinPacker assembly and were slightly better than the results obtained using Trans-ABySS and Trinity assemblers, which indicated that Bridger yielded a more complete transcriptome assembly.

\section{Evaluating coverage and integrity of assembled transcripts using genomic data}

The release of the tea plant genome [15] has provided a reference to assess the accuracy and integrity of transcripts assembled from different assemblers. We first aligned the assembled transcripts to tea plant genomic sequences using BLAT to evaluate the performance of each assembler. The result show that an average of $\sim 98.4 \%$ of the transcripts from five assemblers are mapped (coverage

Table 1 Statistics of the transcriptome assemblies using five representative assemblers with $32 \mathrm{~Gb}$ sequencing data

\begin{tabular}{llllll}
\hline Assembler & $\begin{array}{l}\text { No. } \\
\text { transcript }\end{array}$ & $\begin{array}{l}\text { Total length } \\
(\mathrm{bp})\end{array}$ & $\begin{array}{l}\text { Maximum } \\
\text { transcript } \\
(\mathrm{bp})\end{array}$ & $\begin{array}{l}\text { Average } \\
\text { length } \\
(\mathrm{bp})\end{array}$ & $\begin{array}{l}\text { N50 } \\
(\mathrm{bp})\end{array}$ \\
\hline BinPacker & 423,768 & $368,682,397$ & 21,031 & 870.01 & 1348 \\
Bridger & 380,605 & $364,039,338$ & 22,041 & 956.48 & 1539 \\
SOAPdenovo & 238,364 & $99,912,984$ & 14,281 & 419.16 & 443 \\
Trans-ABySS & 404,455 & $191,731,319$ & 17,014 & 474.05 & 527 \\
Trinity & 404,125 & $225,905,337$ & 14,565 & 559 & 701 \\
\hline
\end{tabular}

$>50 \%$ ), indicating high mapping rates for the transcriptome assembly (Additional file 1: Table S3).

We further investigated the assembly quality of transcripts at the gene level by aligning the transcripts from different assemblers to the coding sequences (CDS) of the tea plant genome and examined the numbers of reconstructed full-length (FL) genes. We found that the total number of FL transcripts (coverage $\geq 98 \%$ ) reconstructed by different assemblers varied significantly, and ranged from 2581 (SOAPdenovo; 1.08\%) to 45,902 (Bridger; $12.06 \%$ ) with an average of 20,306. Approximately $7.36 \%(31,169)$ of the transcripts from BinPacker assembly are defined as FL (Fig. 2a). This proportion is less than that of Bridger but much larger than those of the other three assemblers. Additionally, we assessed the accuracy of each reconstructed FL transcript under three levels, including identity between $50-80 \%, 80-95 \%$, and over $95 \%$. The results indicate that, of the assemblies from five assemblers, Bridger assembly reconstructed the largest number of FL transcripts $(27,641)$ with high accuracy (identity $\geq 95 \%$ ), followed by BinPacker assembly (19,090), Trinity assembly (9928), TransABySS assembly (5533), and SOAPdenovo assembly (2182) (Fig. 2b). We also examined the mapping rate of assembled transcripts with different coverage and identity thresholds (Additional file 1: Figure S2). This analysis indicates a good performance of Bridger and BinPacker for the construction of full-length transcripts in tea plants.

\section{Assessing the sensitivity of assemblers to genes expressed at different levels}

It is a considerable challenge to reconstruct the lowly expressed gene using most current assemblers because these genes are more likely to be fragmented and/or have assembly errors. To investigate the assembly capabilities of current assemblers for genes expressed at different levels, we separately classified genes assembled from different assemblers into ten equal groups according to their expression levels from low to high and then examined their proportions that can be fully and correctly rebuilt. The results show that, as expected, the proportion of fully recovered genes increased at higher expression levels. About 60\% (from 23 to 76\%) of the fully recovered genes come from the top $10 \%$ of highly expressed transcripts (Fig. 3). Although all five assemblers exhibited a poor performance for low expression gene reconstruction, the BinPacker and Bridger assemblers still possessed a high amount of full-length transcripts (Fig. 2a). All five assemblers performed well for highly expressed transcripts (up 50\% quantiles) with an average construction rate of more than $52 \%$. In particular, Bridger assembly harvests the largest amount of fulllength transcripts compared to the other four assemblies 

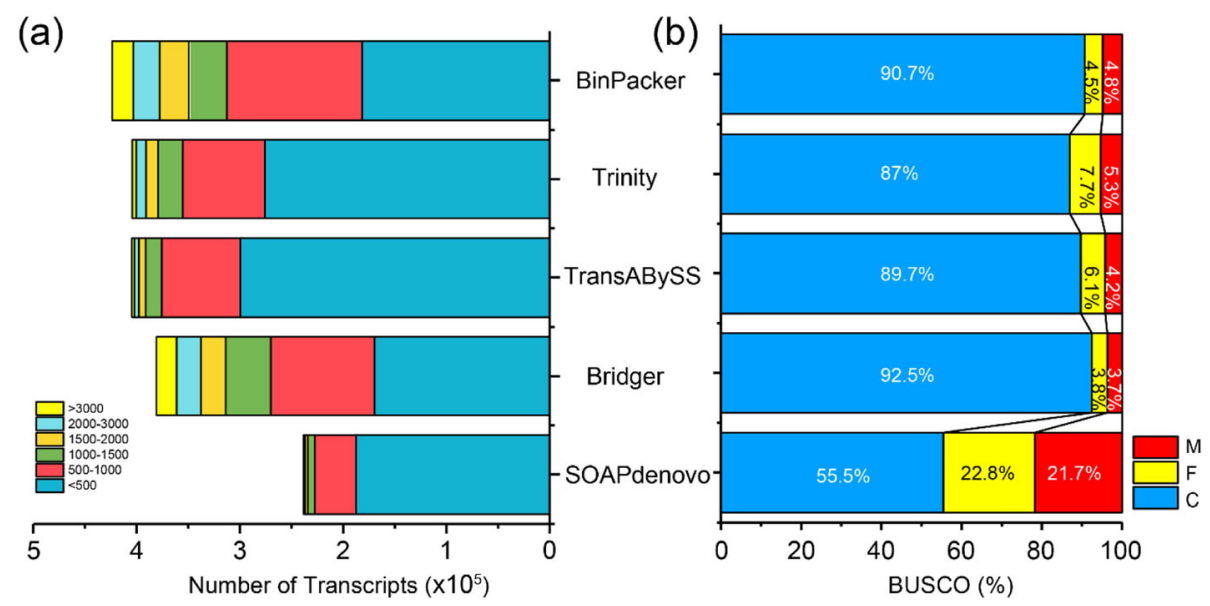

Fig. 1 Overview of the transcriptome assemblies using five state-of-the-art assemblers. a Length distributions. b BUSCO completeness assessment. M: Missing BUSCOs; F: Fragmented BUSCOs; C: Complete BUSCOs

on average, indicating that Bridger is highly capable of tea plant transcriptome assembly. We evaluated the assembly performance for lowly expressed $($ FPKM $<0.1)$ transcripts according to the suggestions from previous study [52]. We found that only a small proportion of lowly expressed transcripts assembled from Bridger (11.1\%), BinPacker (12.5\%), SOAPdenovo (6.7\%), TransABySS (10.3\%), and Trinity (13.3\%) supported by evidence of: 1) read depth $>2$; 2) genome mapping rate > $50 \%$; 3) CDS mapping rate $>60 \%$; and 4 ) NR annotations (Additional file 1: Figure S3). This finding suggests the limitations of the current de novo transcriptome assemblers on the lowly expressed transcripts of tea plants, which is similar to those found in other plant species [53-55]. Nevertheless, the assembly accuracy of BinPacker, Bridger and Trinity are comparable and much higher than that of SOAPdenovo and Trans-ABySS regarding the construction of lowly expressed transcripts. In addition to lowly expressed transcripts, we also investigated the supported evidence of sequence alignment and NR annotation of transcripts with expression levels $\geq 0.1$. These transcripts were divided into three groups: 1) $0.1 \leq \mathrm{FPKM}<1$, 2) $1 \leq \mathrm{FPKM}<5$, and 3) FPKM $\geq 5$. The results show that the quality of the assembled transcripts increased with increasing expression levels (Additional file 1: Figure S3). Compared to the other three assemblers, Bridger and BinPacker showed better performance of transcript assembly not only for weakly but also highly expressed transcripts. Furthermore, we examined the completeness and integrity of the assembled transcripts at different expression levels. We found that highly expressed transcripts exhibit higher assembly completeness and integrity than weakly expressed transcripts (Additional file 1: Figure S4). It is not surprising that weakly expressed transcripts are always truncated and shorted sequences that usually lack sufficient sequencing reads and evidence during transcriptome reconstruction.

\section{Selection of $\boldsymbol{k}$-mer value}

De Bruijn graph is the most commonly used algorithm among the current RNA-seq assemblers, of which $k$-mer is an important parameter that influences the assembly accuracy and efficiency. According to the assessment we conducted, Bridger shows the best performance for tea plant transcriptome assembly. To examine the most suitable $k$-mer values for this assembler, we further investigated the assembly quality by changing $k$-mer values from 19 to 32 (Table 2). The results showed an increase in the total number of assembled transcripts and decreases in both maximum sequence length and N50 with higher $k$-mer values (Additional file 1: Table S4). The results from $k=21$ yielded the longest transcript and maximum N50 size, but it had a high miss rate compared to results from other $k$-mer values. The assemblies from $k=25$ and 27 exhibit a comparable assembly completeness with the lowest missing rate of $3.7 \%$ among all $k$ mer values; however, the results from $k=25$ harvests the longest transcript length and N50 size, which are better than the assembly from $27 k$-mers (Table 2 ). This outcome indicates that a $k$-mer value of 25 is the most suitable for tea plant transcriptome assembly using Bridger.

\section{Optimal data architecture for de novo assembly of tea plant transcriptome}

Tea plants harbor a large and structurally complex genome $[15,16]$. It has not been determined how much sequencing data are actually needed to fully capture the nature of its transcription. To investigate this possibility, we simulated a total of 21 datasets from eight representative tissues of tea plants with a total data amount ranging from 4 to $84 \mathrm{~Gb}$. Each dataset was a mixture of 

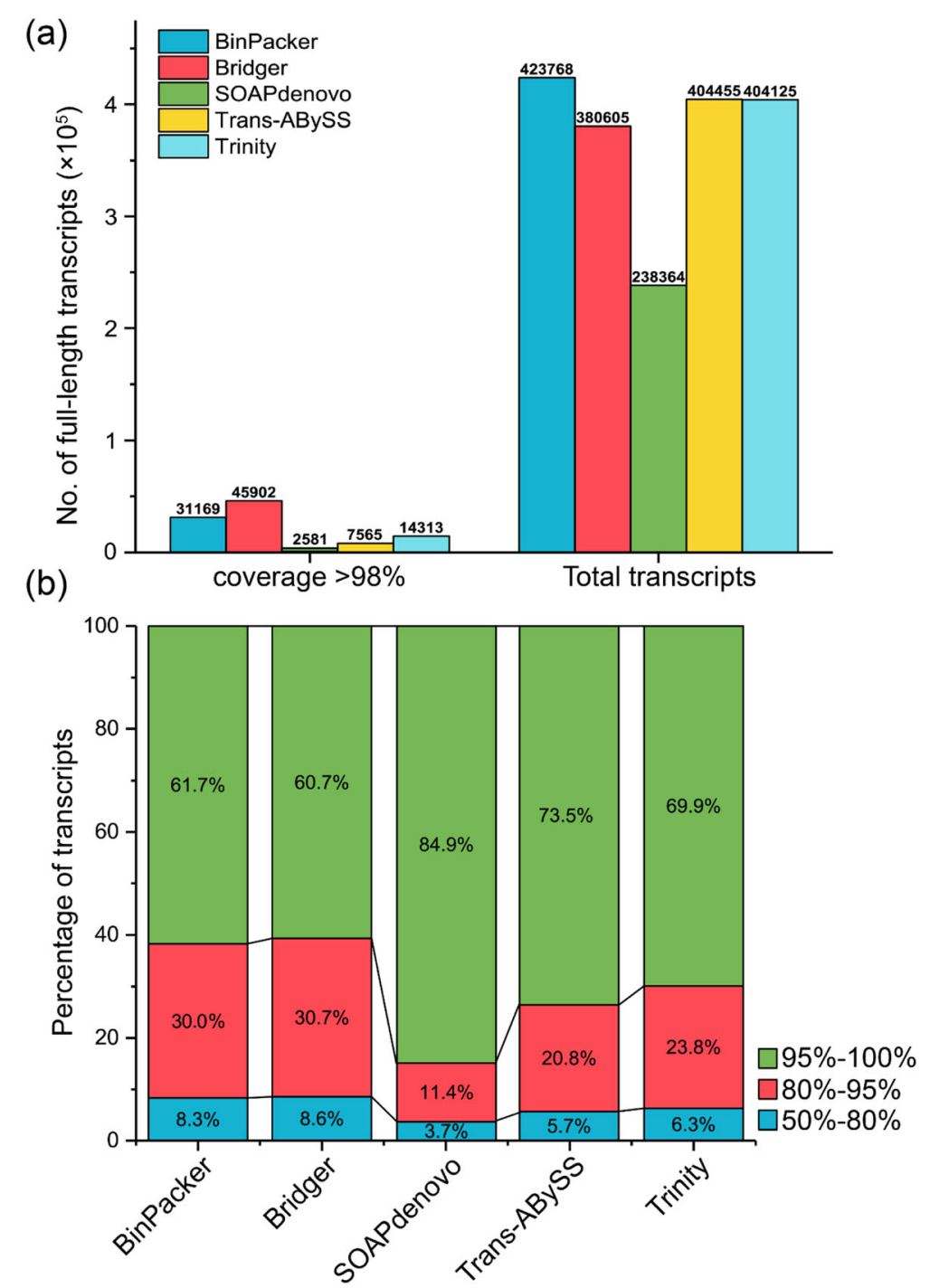

Fig. 2 Quality evaluation of transcriptome assemblies using genome alignment. a The total number of constructed full-length transcripts. $\mathbf{b}$ Percentage of the transcripts with sequence identity $\geq 50 \%$

paired reads randomly extracted from sequencing data of eight tissues. We applied Bridger to assemble the datasets from each data group. The results show that, although the total number of transcripts increased with higher amounts of data, the N50 size and average sequence length of transcripts trended towards stability at $48 \mathrm{~Gb}$ (Fig. 4a). This finding indicates that sequencing data of $48 \mathrm{~Gb}$ may have reached the saturation point for tea plant transcriptome assembly. BUSCO evaluation of the completeness of transcriptome assembly further confirms the assumption. We show that the missing rate of the assembled transcriptome largely decreased from 4 to $20 \mathrm{~Gb}$, then slowly declined, and finally stabilized at 48 $\mathrm{Gb}$ with a missing rate of $2.6 \%$ (Fig. $4 \mathrm{~b}$ ). We resimulated 21 datasets using the same method described above and separately assembled them into transcripts using Bridger, showing a comparable assembly performance and sequence patterns with those reported above. This finding indicates a high repeatability of the obtained results (Additional file 1: Figures S5 and S6).

Tea plants are leaf-use crops of economic importance. In some cases, we merely focus on the transcriptomic dynamics of a few specific tissues or developmental stages. We simulated 88 datasets from eight tissues, 22 datasets from two tissues, and 11 datasets from three tissues (Additional file 1: Table S5 S9). The results indicate that the amount of input reads increases, the number of transcripts obtained by the assembly also increased, and the same phenomenon occurred both in single tissue de novo and multiple polled tissues. At the same input reads, assembling different tissue types will yield different numbers of transcripts. For example, at $1 \mathrm{~Gb}$ reads input, de novo 


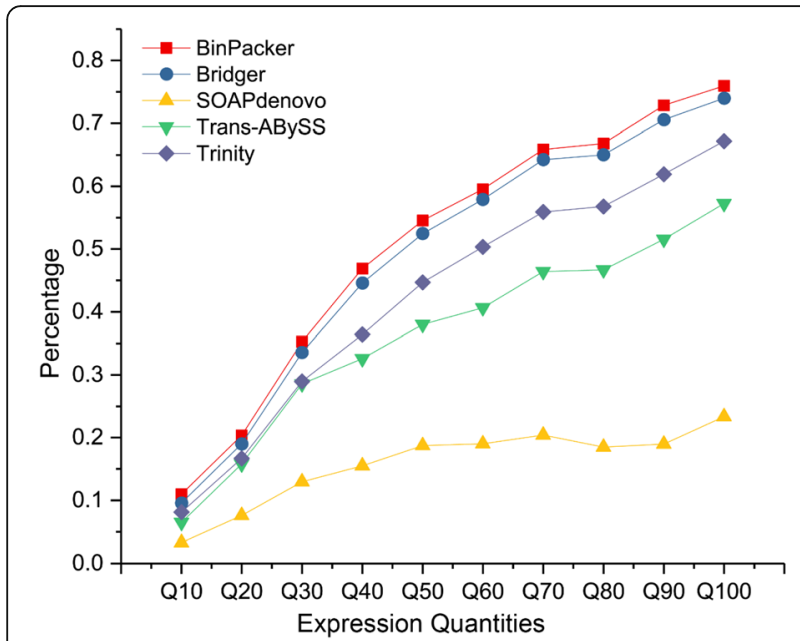

Fig. 3 Full-length genes reconstructed by each assembler at different expression quintiles

bud, root, stem and fruit can provide almost 80,000 transcripts, but the mature leaf in summer yields less than 70 , 000 transcripts. Additionally, the BUSCO evaluation of 1 Gb de novo flower and root both lost over $50 \%$ of BUSCOs. When the input reads were doubled, the missing BUSCO percentage decreased slightly, and the missing rate of the four tissues was lower than $30 \%$. When the input reads reached $6 \mathrm{~Gb}$, the percentage of missing BUSCOs of four tissues was lower than $15 \%$, and that for six of eight tissues was lower than $20 \%$. If we continued to place more reads into the de novo assembly, the percentage of missing BUSCOs became lower in all eight tissues, and was less than $20 \%$ with $9 \mathrm{~Gb}$ reads input while the percentage of missing BUSCO about the first young leaf was less than $10 \%$. When we mixed reads from two tissues or three tissues, we obtained better results compared to the same depth in single tissue de novo assembly (Additional file 1: Table S8 and S9).

Table 2 Summary of the Bridger assemblies using different $k$ mer values

\begin{tabular}{|c|c|c|c|c|c|c|c|}
\hline \multirow{2}{*}{$\begin{array}{l}k- \\
\text { mer }\end{array}$} & \multirow{2}{*}{$\begin{array}{l}\text { No. } \\
\text { transcripts }\end{array}$} & \multirow{2}{*}{$\begin{array}{l}\text { Maximum } \\
\text { length } \\
\text { (bp) }\end{array}$} & \multirow{2}{*}{$\begin{array}{l}\text { Average } \\
\text { length } \\
\text { (bp) }\end{array}$} & \multirow{2}{*}{$\begin{array}{l}\text { N50 } \\
\text { (bp) }\end{array}$} & \multicolumn{3}{|l|}{$\mathrm{BUSCO}^{\mathrm{a}}$} \\
\hline & & & & & $C$ & $\mathrm{~F}$ & $M$ \\
\hline 19 & 273,893 & 27,833 & 1003.51 & 1531 & $56.10 \%$ & $22.30 \%$ & $21.60 \%$ \\
\hline 21 & 350,330 & 28,766 & 987.98 & 1554 & $86.70 \%$ & $6.70 \%$ & $6.60 \%$ \\
\hline 23 & 370,677 & 18,579 & 948.81 & 1524 & $91.20 \%$ & $4.40 \%$ & $4.40 \%$ \\
\hline 25 & 380,605 & 22,041 & 956.48 & 1539 & $92.50 \%$ & $3.80 \%$ & $3.70 \%$ \\
\hline 2 & 383,138 & 20,166 & 951.48 & 1524 & $93.00 \%$ & $3.30 \%$ & $3.70 \%$ \\
\hline 29 & 383,689 & 19,347 & 950.33 & 1513 & $86.70 \%$ & $6.70 \%$ & $6.60 \%$ \\
\hline 32 & 390,761 & 19,996 & 896.6 & 1430 & $92.70 \%$ & $3.30 \%$ & $4.00 \%$ \\
\hline
\end{tabular}

${ }^{\mathrm{a}} \mathrm{M}$ Missing BUSCOs, F Fragmented BUSCOs, C Complete BUSCOs

\section{Time usage by different assemblers}

The run times required for each assembler to assemble the same amount of input reads vary significantly. A suitable assembly software not only performs well in assembly quality, but also should assemble in the least time possible. We simulated three data sets $(0.5 \mathrm{~Gb}, 1.0$ $\mathrm{Gb}, 3 \mathrm{~Gb})$ to compare the run times of five assemblers with different $k$-mer values $(19,21,23,25,27,29,32)$. The $k$-mer value of Trinity is fixed to 25 . We analyzed the results of run time requirements, and found large differences among the tested assembly software (Additional file 1: Table S10). Taking $k=25$ as an example, SOAPdenovo had the shortest assembly time, followed by Trans-ABySS. The other three assembly software required considerably more time than Trans-ABySS, with Trinity requiring the most. With an increased number of reads, the assembly time of each software also increased, though it was not by multiples as reads increased. Another phenomenon is that when the $k$ was set to 19 , assembly times were considerably longer than with other $k$-mer values.

\section{Discussion}

De novo assembly of transcriptomes from short-read RNA-Seq data presents several challenges to bioinformaticians. This study was designed to evaluate the performances of five publicly available assemblers that are commonly used to assemble short-read transcriptome data: SOAPdenovo, Trans-ABySS, Bridger, BinPacker and Trinity. To reveal the key factors to consider when choosing an optimal strategy and software tool, we set up variable testing conditions by using five assemblers using the same input reads and with single $k$-mer (25) and multiple $k$-mer (19 32). The assemblers were compared under these conditions while mapping to such factors as genome, CDS, and low coverage depth vs. high coverage depth. We measured results in terms of run time usage, transcript accuracy, integrity and completeness, and sensitivity to assembling transcripts from low to high expression levels. By analyzing and comparing the assembled results under various conditions, we were able to develop useful guidelines that may facilitate future transcriptome studies.

We found that the performances of Bridger and BinPacker were better than those of the other three assemblers, at least in the tea plant transcriptome assembly. Currently, advances in third-generation sequencing technology will accelerate the transcriptome study of several crop species $[56,57]$. Accordingly, the completeness of the transcriptome generated from third-generation sequencing (e.g., PacBio SMRT) can be used as a key factor to evaluate the assembly performance of the transcriptome from nextgeneration sequencing. Two transcript datasets of tea plants were recently generated using PacBio SMRT sequencing 

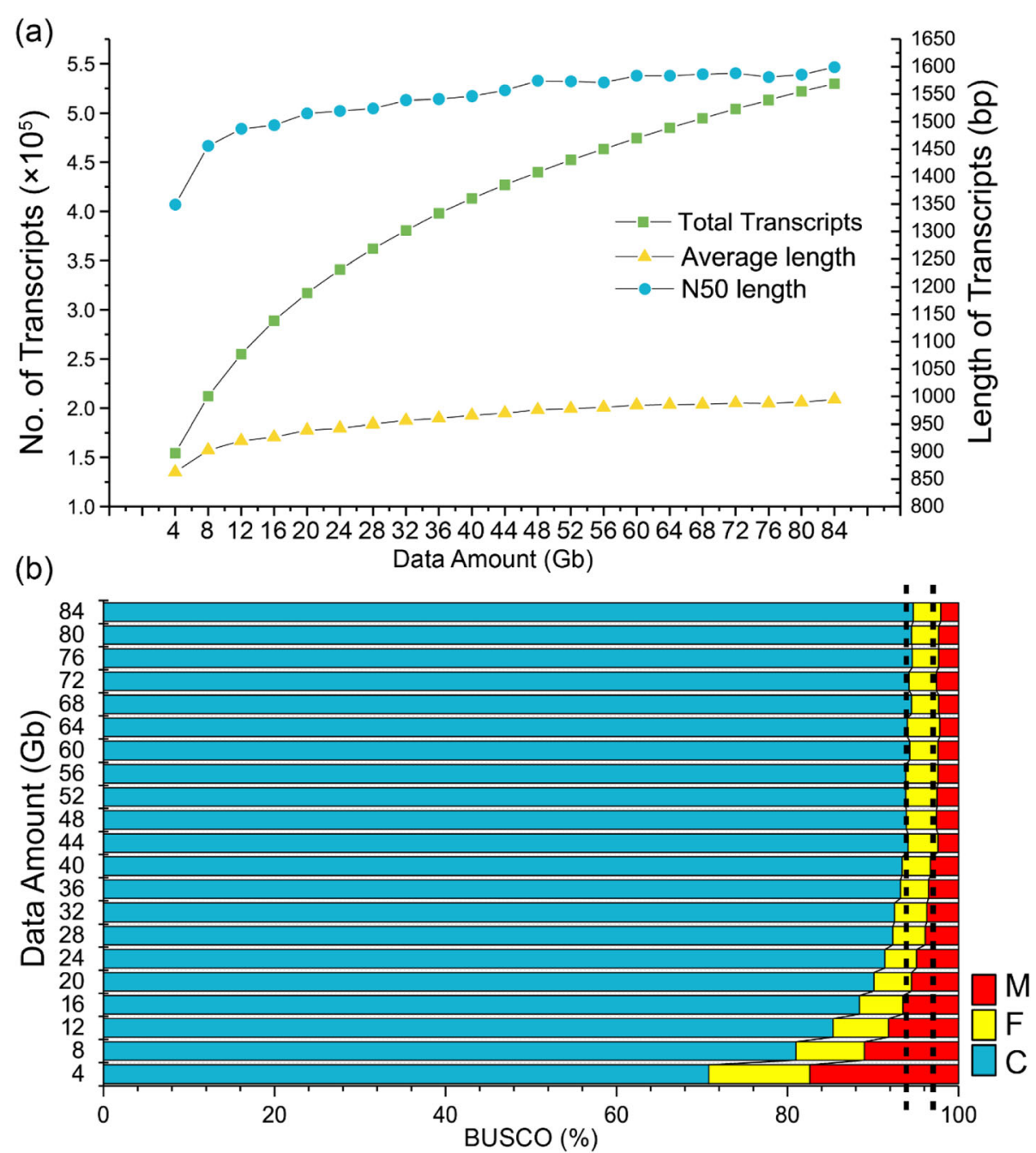

Fig. 4 Statistic of the transcriptome assemblies using Bridger with different amount of sequencing data from replicate 1. a Total number and sequence length of the transcripts. The x-axis represents the total amount of the sequencing data ranging from 4 to 84 Gb; b BUSCO evaluation of the completeness of transcriptome assemblies. The $x$-axis indicates the percentage of each type of BUSCO, while the $y$-axis displays the transcriptome assembled using different amount of sequencing data. M: Missing BUSCOs; F: Fragmented BUSCOs; C: Complete BUSCOs

technology $[3,58]$. They contain 80,217 and 93,833 transcripts, respectively. Analysis of their completeness using plant BUSCOs found that the completeness of these two transcripts datasets of tea plant generated using thirdgeneration sequencing varied significantly, ranging from 55.1 to $92.7 \%$ (Additional file 1: Table S11), with the best performance showing a similar completeness of our NGS transcriptome assembly (92.5\%) with $32 \mathrm{~Gb}$ data. This result indicates that transcriptome assembled from sufficient NGS sequencing data (e.g., $32 \mathrm{~Gb}$ or more) can achieve a comparable completeness of transcriptome from thirdgeneration sequencing technology.

In addition, although polyploidy is widespread in angiosperms, most of the current transcriptome assemblers are typically developed, refined, and tested for diploid organisms [59-64]. De novo assembly of the transcriptome of diploid plants is notably easy using the currently available assemblers. However, this approach has several unique challenges in the transcriptome assembly of polyploidy plants particularly with the large genome size, which possess a large number of paralogues, orthologues, homologues and isoforms that are difficult to disentangle at the sequence level [65-67]. All of the assembler assessments and parameter tests in the present study are optimal for diploid plants (e.g., tea plants). However in the case of better constructing the transcriptome of polyploidy plants, we believe that future research is warranted to 1) prepare high-quality material and extract high-quality nonfragmented RNA; 2) obtain adequate depth of sequencing reads with high-quality and long sequence length (e.g., PacBio SMRT); and (3) develop suitable software/pipeline that can efficiently discriminate homologue nucleotide 
differences and errors among the sequencing data. This research may eventually help to resolve the transcriptome complexity of polyploidy plants $[68,69]$.

It is widely accepted that most plants (e.g., tea plants) have undergone multiple whole genome duplication events that accordingly duplicate their protein-coding genes [70, 71]. This largely challenged the duplicated genes (transcripts) construction with the current transcriptome assemblers, as almost all of the assemblers are difficult to distinguish the origin of duplicated transcripts at the sequence level (e.g., PCR amplification, assembly redundancy, alternative splicing or even assembly errors) $[72,73]$. In the present study, we used the BUSCO pipeline to evaluate the proportion of duplicated transcripts recovered by different assemblers. The results show that approximately $40 \%$ of duplicated genes were moderately constructed by the five assemblers. Compared to the other three assemblers, Bridger and BinPacker showed high performance in duplicate gene reconstruction. Transcripts with short or long sizes also presents considerably huge difficulties to the current de novo transcriptome assemblers. The short transcripts are always truncated and weakly expressed, as they usually lack sufficient sequencing reads and evidence for transcript reconstruction. As reported previously $[17,44]$, the annotation efficiency of the NR database was negatively related to the length of assembled transcripts. For example, over $80 \%$ of transcripts are not able to find any homologues in the NR database if their sequence length is $<500 \mathrm{bp}$. Similar situations also occurred in the transcripts with long sequence lengths. The long transcripts may be derived from the overassembly of nearby transcripts. In particular, this situation is highly common in the transcriptome assembly of fungal species, as most of the fungal species always have high gene density $[74,75]$. For plant species, the average proportion of long transcripts $(>5 \mathrm{~kb})$ from transcriptome assembly is approximately $2 \%$ [14]. Most of these transcripts $(\sim 60 \%)$ are confirmed to be aligned with multiple homologues of the NR database, showing incorrect linking during the assembly. Therefore, most of the current de novo assemblers may possess poor abilities to resolve the duplicated transcripts, as well as some notably short or long transcripts, which should receive more attention in transcriptome studies in the future.

\section{Conclusions}

In this study, we comprehensively evaluated the de novo transcriptomic assemblies of tea plants with different amounts of RNA-seq data employing five well-known assemblers. Although the total number of assembled transcripts increased with the growing amount of sequencing data, the proportion of unassembled transcripts became saturated as revealed by plant BUSCO datasets. The assembler Bridger and BinPacker performed better in the tea plant transcriptome assembly, which also harbored the shorter running time. Nevertheless, the Bridger package shows the best performance in both assembly completeness and accuracy, as evaluated using the BUSCO datasets and genomic alignments. This study will be of significance in helping the tea research community gain better sequencing and assembly of tea plant transcriptomes under conditions of interest, and thus help to answer the major biological questions currently facing the tea industry.

\section{Materials and methods \\ Plant material}

The 6-year-old tea plant (C. sinensis var. sinensis cv. shuchazao) used in this study were planted in the DeChang Tea Plantation of Anhui, China. Eight tissues, including apical bud, first young leaf, second young leaf, mature leaf in summer, stem, flower, fruit, and root were sampled and stored at $-80^{\circ} \mathrm{C}$.

\section{RNA isolation, transcriptomic library construction and RNA sequencing}

As described in a previous study [51], total RNA was extracted separately from the 8 tissues using a modified $\mathrm{CTAB}$ method in triplicate. The yield and quality of RNA were determined by agarose gel electrophoresis and by measuring with a Nanodrop 2000. Equal amounts of RNA from three different samples were pooled before cDNA library preparation. The mRNA enrichment, cDNA synthesis, fragmentation, adapter addition, selection of fragment size, PCR amplification, and transcriptomic sequencing were performed according to the manufacturer's protocol (Illumina, CA, USA). The cDNA library was examined using an Agilent 2100 Bioanalyzer prior to sequencing on an Illumina HiSeq 2000 platform with a read length of $90 \mathrm{bp}$.

\section{Data preprocessing and de novo assembly}

Preliminary screening was performed on raw sequencing reads to remove low-quality reads and reads with adaptor sequences using the Trimmomatic (version 0.32) program [76]. Only reads with a trimmed length over $30 \mathrm{bp}$ were used in further analyses. The number of paired-end reads in each sample is shown in Additional file 1: Table S2. The remaining reads were then adopted for de novo assembly using the following assemblers with default parameters: Trinity (release-20,130,225) [6], Bridger (201412-01) [7], SOAPdenovo (ver. 1.04) [8], Trans-ABySS (ver. 1.55) [9], and BinPacker (ver. 1.0) [10].

The simulated data of different sequencing depths were subsampled from the real data derived from the 8 samples using a Perl script. We tested four de novo models: the first is to assemble $32 \mathrm{~Gb}$ reads by randomly choosing 4 $\mathrm{Gb}$ per tissue; the second use simulation method to generate $4 \mathrm{~Gb}(0.5 \mathrm{~Gb}$ per tissue) to $84 \mathrm{~Gb}(10.5 \mathrm{~Gb}$ per tissue) 
reads. The third model is to assemble transcripts from 1 to $11 \mathrm{~Gb}$ (1 Gb per step) reads per sample. The fourth model is to assemble two or three samples from different tissue combinations, such as one bud and leaf, one leaf and root, one bud and two leaves.

\section{Mapping reconstructed transcripts to genomic and coding sequences}

Genome sequence and gene models for tea plants were downloaded from the Tea Plant Information Archive (http://tpia.teaplant.org), and used to evaluate the performance of each assembler [77]. BLAT [78] with default parameters was applied to map the reconstructed transcripts from each assembler to non-identical reference coding sequences and reference genomes. Four groups of hits were classified to evaluate the capability for CDS reconstruction: 1) Coverage of the entire reference coding sequence without mismatches, insertions or deletions (98\%); 2, 3, 4) At least $95 \% / 80 \% / 50 \%$ of sequence alignment identity covering the entire reference coding sequence. To assess the accuracy of reconstructed transcripts, we also aligned them to the reference genome using BLAT, and accuracy was assessed using the numbers of reconstructed transcripts that had at least $50 \%$ of their lengths aligned back to genome. Transcripts with less than $50 \%$ of their lengths mapped back to the genome were defined as unmapped transcripts. The Tbtools [79] software was used to plot the graphical heat map.

\section{Evaluation of assembly quality using BUSCO}

For further quantitative assessment of the assembly completeness, we applied BUSCO [80] with default settings. The BUSCO tool analyzed each transcriptome assembly state with complete and single-copy BUSCOs, complete and duplicated BUSCOs, fragmented BUSCOs, and missing BUSCOs using the embryophyta_odb9 database, which contains 1440 total BUSCO groups.

\section{Calculation of gene expression}

The expression levels of transcripts were calculated using the FPKM method. First, reads were mapped to transcript datasets using Bowtie2 (version 2.1.0) [81] in a sensitive setting, and then FPKM values for each transcripts were subsequently calculated by RESM (version 1.2.29) [82].

\section{Supplementary information}

Supplementary information accompanies this paper at https://doi.org/10. 1186/s12859-019-3166-x.

Additional file 1: Figure S1. Venn diagram shows the transcripts intersected in different assemblers. Figure S2. Transcripts mapped to coding sequences and genome sequences with different coverage and identity thresholds. Figure S3. Assembly quality of transcripts at different expression levels. Figure $\mathbf{S 4}$. Completeness of the assembled transcripts at different expression levels. Figure S5. Comparison of the assembly performances between two replications of datasets randomly selected from eight representative tissues of tea plant. Figure S6. Statistic of the transcriptome assemblies using Bridger with different amount of sequencing data from replicate 2. Table S1. Summary of transcriptome assemblies of tea plant in previous studies. Table S2. Summary of the data used in this study. Table S3. Coverage of transcripts mapped to the reference genome. Table S4. Statistic of the Bridger assembly using different $k$-mer values. (a) Assembly characteristics; (b) completeness assessment using BUSCO; (c) length distribution. Table S5. Statistics of assembly. (a) Apical bud; (b) flower; (c) fruit; (d) second young leaf; (e) mature leaf in summer; (f) first young leaf; (g) root; (h) stem. Table S6. Length distribution of assembly. (a) Apical bud; (b) flower; (c) fruit, (d) second young leaf; (e) mature leaf in summer; (f) first young leaf; (g) root; (h) stem. Table S7. BUSCO evaluation of assembly. (a) Apical bud; (b) flower; (c) fruit; (d) second young leaf; (e) mature leaf in summer; (f) first young leaf; (g) root; (h) stem. Table S8. Statistic of assembly. (a) Apical bud and first young leaf; (b) apical bud and root. Table S9. Statistic of pooled assembly. (a) Assembly characteristics; (b) length distribution; (c) BUSCO evaluation. Table S10. Runtime (hours) performance for each assembler with different amount of input data. (a) 0.5 Gb; (b) 1 Gb; (c) 3 Gb. Table S11. Completeness of transcriptomes generated in tea plant using PacBio technology.

\section{Abbreviations}

BUSCO: Benchmarking universal single-copy orthologs; CDS: Coding sequence; FL: Full length; RNA-seq: RNA sequencing

\section{Authors' contributions}

C W and E X conceptualized and designed the study. FL and WT analyzed the data and drafted the manuscript. All authors have read and approved the final manuscript.

\section{Funding}

This work was supported by the National Key Research and Development Program of China (2018YFD1000601), the National Natural Science Foundation of China (31800180; 31171608), the Natural Science Foundation of Anhui Province (1908085MC75), the Special Innovative Province

Construction in Anhui Province (15czs08032), the Special Project for Central Guiding Science and Technology Innovation of Region in Anhui Province (2016080503B024) and the Programme for Changjiang Scholars and Innovative Research Team in University (IRT1101).

Availability of data and materials

The Illumina RNA-seq data is available in the NCBI SRA database under the accessions of SRR1928149 and SRP056466.

Ethics approval and consent to participate Not applicable

\section{Consent for publication}

Not applicable

\section{Competing interests}

The authors declare that they have no competing interests.

\section{Author details}

${ }^{1}$ State Key Laboratory of Tea Plant Biology and Utilization, Anhui Agricultural University, Hefei 230036, China. ${ }^{2}$ School of Science, Anhui Agricultural

University, Hefei 230036, China.

Received: 23 April 2019 Accepted: 21 October 2019

Published online: 06 November 2019

\section{References}

1. Yuichi K, Martin S, Rasko L. The sequence Read archive: explosive growth of sequencing data. Nucleic Acids Res. 2012;40(Database issue):54-6.

2. Kumar R, Ichihashi Y, Kimura S, Chitwood DH, Headland LR, Peng J, Maloof JN, Sinha NR. A high-throughput method for Illumina RNA-Seg library preparation. Front Plant Sci. 2012;3:202. 
3. Xu Q, Zhu J, Zhao S, Hou Y, Li F, Tai Y, Wan X, Wei C. Transcriptome profiling using single-molecule direct RNA sequencing approach for indepth understanding of genes in secondary metabolism pathways of Camellia sinensis. Front Plant Sci. 2017:8:1205.

4. Blencowe BJ, Ahmad S, Lee LJ. Current-generation high-throughput sequencing: deepening insights into mammalian transcriptomes. Genes Dev. 2009;23(12):1379.

5. Oshlack A, Robinson MD, Young MD. From RNA-seq reads to differential expression results. Genome Biol. 2010;11(12):220.

6. Grabherr MG, Haas BJ, Yassour M, Levin JZ, Thompson DA, Amit I, Xian A, Fan L, Raychowdhury R, Zeng Q. Trinity: reconstructing a full-length transcriptome without a genome from RNA-Seq data. Nat Biotechnol. 2013;29(7):644.

7. Zheng C, Li G, Liu J, Yu Z, Ashby C, Liu D, Cramer CL, Huang X. Bridger: a new framework for de novo transcriptome assembly using RNA-seq data. Genome Biol. 2015;16(1):30

8. Xie Y, Wu G, Tang J, Luo R, Patterson J, Liu S, Huang W, He G, Gu S, Li S. SOAPdenovo-trans: de novo transcriptome assembly with short RNA-Seq reads. Bioinformatics. 2014;30(12):1660-6.

9. Simpson JT, Wong K, Jackman SD, Schein JE, Jones SJ, Birol I. ABySS: a parallel assembler for short read sequence data. Genome Res. 2009;19(6):1117.

10. Liu J, Zhu Y, Luo GZ, Wang X, Yue Y, Wang X, Zong X, Chen K, Yin H, Fu Y, et al. Abundant DNA $6 \mathrm{~mA}$ methylation during early embryogenesis of zebrafish and pig. Nat Commun. 2016;7:13052.

11. Trapnell C, Williams BA, Pertea G, Mortazavi A, Kwan G, Baren MJV, Salzberg SL, Wold BJ, Pachter L. Transcript assembly and abundance estimation from RNA-Seq reveals thousands of new transcripts and switching among isoforms. Nat Biotechnol. 2010;28(5):511-5.

12. Willson KC, Clifford MN. Tea: cultivation to consumption. Ecol Freshw Fish. 1992;5(4):175-82.

13. Mondal TK, Bhattacharya A, Laxmikumaran M, Ahuja PS. Recent advances of tea (Camellia Sinensis) biotechnology. Plant Cell Tissue Organ Culture. 2004;76(3):195-254

14. Li CF, Zhu Y, Yu Y, Zhao QY, Wang SJ, Wang XC, Yao MZ, Luo D, Li X, Chen L. Global transcriptome and gene regulation network for secondary metabolite biosynthesis of tea plant (Camellia sinensis). BMC Genomics. 2015;16(1):560

15. Wei C, Yang H, Wang S, Zhao J, Liu C, Gao L, Xia E, Lu Y, Tai Y, She G. Draft genome sequence of Camellia sinensis var. sinensis provides insights into the evolution of the tea genome and tea quality. Proc Natl Acad Sci U S A. 2018:115(18):201719622.

16. Xia EH, Zhang HB, Sheng J, Li K, Zhang QJ, Kim C, Zhang Y, et al. The tea tree genome provides insights into tea flavor and independent evolution of caffeine biosynthesis. Mol Plant. 2017;10(6):866-77.

17. Chen Q, Li YY, Sun J, Jiang CJ, Zhang ZZ, Yu O, Wei CL, Yang H, Shi CY, Xia T. Deep sequencing of the Camellia sinensis transcriptome revealed candidate genes for major metabolic pathways of tea-specific compounds. BMC Genomics. 2011;12(1):131.

18. Wang XC, Zhao QY, Ma CL, Zhang ZH, Cao HL, Kong YM, Yue C, Hao XY, Chen L, Ma JQ. Global transcriptome profiles of Camellia sinensis during cold acclimation. BMC Genomics. 2013;14(1):415.

19. Dong B, Wu B, Hong W, Li X, Zhuo L, Li X, Huang Y. Transcriptome analysis of the tea oil camellia (Camellia oleifera) reveals candidate drought stress genes. PLoS One. 2017;12(7):e0181835

20. Li Y, Huang J, Song $X$, Zhang Z, Jiang Y, Zhu Y, Zhao H, Ni D. An RNA-Seq transcriptome analysis revealing novel insights into aluminum tolerance and accumulation in tea plant. Planta. 2017;246(13):1-13.

21. Li QS, Lin XM, Qiao RY, Zheng XQ, Lu JL, Ye JH, Liang YR. Effect of fluoride treatment on gene expression in tea plant (Camellia sinensis). Sci Rep. 2017;7(1):9847.

22. Zheng C, Wang Y, Ding Z, Zhao L. Global transcriptional analysis reveals the complex relationship between tea quality, Leaf Senescence and the Responses to Cold-Drought Combined Stress in Camellia sinensis. Front Plant Sci. 2016;7:1858.

23. Zheng C, Zhao L, Wang Y, Shen J, Zhang Y, Jia S, Li Y, Ding Z. Integrated RNA-Seq and sRNA-Seq analysis identifies chilling and freezing responsive key molecular players and pathways in tea plant (Camellia sinensis). PLoS One. 2015;10(4):e0125031.

24. Song L, Ma Q, Zou Z, Sun K, Yao Y, Tao J, Kaleri NA, Li X. Molecular Link between Leaf Coloration and Gene Expression of Flavonoid and Carotenoid Biosynthesis Incamellia sinensiscultivar 'Huangjinya. Front Plant Sci. 2017;8:803.
25. Jayaswall K, Mahajan P, Singh G, Parmar R, Seth R, Raina A, Swarnkar MK, Singh AK, Shankar R, Sharma RK. Transcriptome analysis reveals candidate genes involved in blister blight defense in tea (Camellia sinensis (L) Kuntze). Sci Rep. 2016;6:30412.

26. Wei K, Wang L, Zhang C, Wu L, Li H, Zhang F, Cheng H. Transcriptome analysis reveals key flavonoid 3'-hydroxylase and flavonoid 3',5'-hydroxylase genes in affecting the ratio of Dihydroxylated to Trihydroxylated Catechins in Camellia sinensis. PLoS One. 2015;10(9):e0137925.

27. Liu SC, Jin JQ, Ma JQ, Yao MZ, Ma CL, Li CF, Ding ZT, Chen L. Transcriptomic analysis of tea plant responding to drought stress and recovery. PLoS One. 2016;11(1):e0147306.

28. Hao X, Li L, Hu Y, Chao Z, Wang X, Lu W, Zeng J, Yang Y. Transcriptomic analysis of the effects of three different light treatments on the biosynthesis of characteristic compounds in the tea plant by RNA-Seq. Tree Genet Genomes. 2016;12(6):118.

29. Wang M, Zhang X, Li Q, Chen X, Li X. Comparative transcriptome analysis to elucidate the enhanced thermotolerance of tea plants (Camellia sinensis) treated with exogenous calcium. Planta. 2019.

30. Shi Y-L, Sheng Y-Y, Cai Z-Y, Yang R, Li Q-S, Li X-M, Li D, Guo X-Y, Lu J-L, Ye $\mathrm{J}$-H. Involvement of salicylic acid in anthracnose infection in tea plants revealed by Transcriptome profiling. Int J Mol Sci. 2019;20(10):2439.

31. Liu F, Wang Y, Ding Z, Zhao L, Xiao J, Wang L, Ding S. Transcriptomic analysis of flower development in tea ( Camellia sinensis (L.)). Gene. 2017:631:39.

32. Tan LQ, Wang LY, Wei K, Zhang CC, Wu LY, Qi GN, Cheng H, Zhang Q, Cui QM, Liang JB. Floral Transcriptome sequencing for SSR marker development and linkage map construction in the tea plant (Camellia sinensis). PLoS One 2013;8(11):e81611.

33. Wei $K$, Wang $L Y$, Wu LY, Zhang CC, Li HL, Tan LQ, Cao HL, Cheng H. Transcriptome analysis of indole-3-butyric acid-induced adventitious root formation in nodal cuttings of Camellia sinensis (L.). Plos One. 2014;9(9):e107201

34. Wu ZJ, Li XH, Liu ZW, Xu ZS, Zhuang J. De novo assembly and transcriptome characterization: novel insights into catechins biosynthesis in Camellia sinensis. BMC Plant Biol. 2014;14(1):277.

35. Shi J, Ma C, Qi D, Lv H, Yang T, Peng Q, Chen Z, Lin Z. Transcriptional responses and flavor volatiles biosynthesis in methyl jasmonate-treated tea leaves. BMC Plant Biol. 2015;15(1):233.

36. Li CF, Xu YX, Ma JQ, Jin JQ, Huang DJ, Yao MZ, Ma CL, Chen L. Biochemical and transcriptomic analyses reveal different metabolite biosynthesis profiles among three color and developmental stages in 'Anji Baicha' (Camellia sinensis). BMC Plant Biol. 2016;16(1):195.

37. Wang YN, Tang L, Hou Y, Wang P, Yang H, Wei CL. Differential transcriptome analysis of leaves of tea plant (Camellia sinensis) provides comprehensive insights into the defense responses to Ectropis oblique attack using RNA-Seq. Funct Integr Genomics. 2016;16(4):383-98.

38. Jin JQ, Ma JQ, Yao MZ, Ma CL, Chen L. Functional natural allelic variants of flavonoid $3^{\prime}, 5^{\prime}$-hydroxylase gene governing catechin traits in tea plant and its relatives. Planta. 2016;245(3):1-16.

39. Pan J, Wang W, Li D, Shu Z, Ye X, Chang P, Wang Y. Gene expression profile indicates involvement of $\mathrm{NO}$ in Camellia sinensis pollen tube growth at low temperature. BMC Genomics. 2016;17(1):809.

40. Zhang CC, Wang LY, Wei K, Wu LY, Li HL, Zhang F, Cheng H, Ni DJ. Transcriptome analysis reveals self-incompatibility in the tea plant (Camellia sinensis) might be under gametophytic control. BMC Genomics. 2016;17(1):359.

41. Wei K, Zhang Y, Wu L, Li H, Ruan L, Bai P, Zhang C, Zhang F, Xu L, Wang L. Gene expression analysis of bud and leaf color in tea. Plant Physiol Biochem. 2016;107:310-8.

42. Paul A, Jha A, Bhardwaj S, Singh S, Shankar R, Kumar S. RNA-seq-mediated transcriptome analysis of actively growing and winter dormant shoots identifies non-deciduous habit of evergreen tree tea during winters. Sci Rep. 2014;4:5932.

43. Ono E, Handa T, Koeduka T, Toyonaga H, Tawfik MM, Shiraishi A, Murata J, Matsui K. CYP74B24 is the 13-hydroperoxide lyase involved in biosynthesis of green leaf volatiles in tea (Camellia sinensis). Plant Physiol Biochem Ppb. 2016;98:112-8.

44. Zhang HB, Xia EH, Huang H, Jiang JJ, Liu BY, Gao LZ. De novo transcriptome assembly of the wild relative of tea tree (Camellia taliensis) and comparative analysis with tea transcriptome identified putative genes associated with tea quality and stress response. BMC Genomics. 2015;16(1):1-14. 
45. Wu Q, Chen Z, Sun W, Deng T, Chen M. De novoSequencing of the leaf Transcriptome reveals complex light-responsive regulatory networks inCamellia sinensiscv. Baijiguan. Front Plant Sci. 2016;7.

46. Chen C, Wei K, Wang L, Ruan L, Li H, Zhou X, Lin Z, Shan R, Cheng H. Expression of key structural genes of the phenylpropanoid pathway associated with catechin epimerization in tea cultivars. Front Plant Sci. 2017;8:702.

47. Li W, Xiang F, Zhong M, Zhou L, Liu H, Li S, Wang X. Transcriptome and metabolite analysis identifies nitrogen utilization genes in tea plant (Camellia sinensis). Sci Rep. 2017;7(1):1693.

48. Li NN, Yue C, Cao HL, Qian WJ, Hao XY, Wang YC, Wang L, Ding CQ, Wang $X C$, Yang YJ. Transcriptome sequencing dissection of the mechanisms underlying differential cold sensitivity in young and mature leaves of the tea plant ( Camellia sinensis ). J Plant Physiol. 2018;224-225:144-55.

49. Yang H, Wang Y, Li L, Li F, He Y, Wu J, Wei C. Transcriptomic and phytochemical analyses reveal root-mediated resource-based defense response to leaf Herbivory by Ectropis oblique in tea plant (Camellia sinensis). J Agric Food Chem. 2019;67(19):5465-76.

50. Zhao QY, Wang Y, Kong YM, Luo D, Li X, Hao P. Optimizing de novo transcriptome assembly from short-read RNA-Seq data: a comparative study. BMC Bioinformatics. 2011;12(14):S2.

51. Tai Y, Chun L, Shuwei Y, Hua Y, Jiameng S, Chunxiao G, Bei H, Zhaoye L, Yi $Y$, Enhua X. Gene co-expression network analysis reveals coordinated regulation of three characteristic secondary biosynthetic pathways in tea plant (Camellia sinensis). Bmc Genomics. 2018;19(1):616.

52. Chen S, Ren C, Zhai J, Yu J, Zhao X, Li Z, Zhang T, Ma W, Han Z, Ma C. CAFU: a galaxy framework for exploring unmapped RNA-Seq data. Brief Bioinform. 2019.

53. Harikrishnan SL, Pucholt P, Berlin S. Sequence and gene expression evolution of paralogous genes in willows. Sci Rep. 2015;5:18662.

54. Ranjan A, Ichihashi Y, Farhi M, Zumstein K, Townsley B, David-Schwartz R, Sinha NR. De novo assembly and characterization of the transcriptome of the parasitic weed dodder identifies genes associated with plant parasitism. Plant Physiol. 2014;166(3):1186-99.

55. Ryder N, Dorn KM, Huitsing M, Adams M, Ploegstra J, DeHaan L, Larson S, Tintle NL. Transcriptome assembly and annotation of johnsongrass (Sorghum halepense) rhizomes identify candidate rhizome-specific genes. Plant Direct. 2018;2(6):e00065.

56. Wang B, Tseng E, Regulski M, Clark TA, Hon T, Jiao Y, Lu Z, Olson A, Stein JC, Ware $\mathrm{D}$. Unveiling the complexity of the maize transcriptome by singlemolecule long-read sequencing. Nat Commun. 2016;7:11708.

57. Zhang G, Sun M, Wang J, Lei M, Li C, Zhao D, Huang J, Li W, Li S, Li J. PacBio full-length CDNA sequencing integrated with RNA-seq reads drastically improves the discovery of splicing transcripts in rice. Plant $\mathrm{J}$. 2019;97(2):296-305.

58. Qiao D, Yang C, Chen J, Guo Y, Li Y, Niu S, Cao K, Chen Z. Comprehensive identification of the full-length transcripts and alternative splicing related to the secondary metabolism pathways in the tea plant (Camellia sinensis). Sci Rep. 2019;9(1):2709.

59. Liu T, Zhu S, Tang Q, Chen P, Yu Y, Tang S. De novo assembly and characterization of transcriptome using Illumina paired-end sequencing and identification of CesA gene in ramie ( Boehmeria nivea L. gaud ). BMC Genomics. 2013;14(1):125.

60. Garg R, Patel RK, Tyagi AK, Jain M. De novo assembly of chickpea transcriptome using short reads for gene discovery and marker identification. DNA Research,18,1(2011-1-7). 2011;18(1):53-63.

61. Kim JE, Choe J, Lee WK, Kim S, Lee MH, Kim TH, Jo SH, Lee JH. De novo gene set assembly of the transcriptome of diploid, oilseed-crop species Perilla citriodora. J Plant Biotechnol. 2016;43(3):293-301.

62. Fox SE, Geniza M, Hanumappa M, Naithani S, Sullivan C, Preece J, Tiwari VK, Elser J, Leonard JM, Sage A. De Novo Transcriptome Assembly and Analyses of Gene Expression during Photomorphogenesis in Diploid Wheat Triticum monococcum. PLoS ONE. 2014;9(5):e96855.

63. Li X, Acharya A, Farmer AD, Crow JA, Bharti AK, Kramer RS, Wei Y, Han Y, Gou J, May GD. Prevalence of single nucleotide polymorphism among 27 diverse alfalfa genotypes as assessed by transcriptome sequencing. BMC Genomics. 2012;13(1):568.

64. Ni L, Ren X, Xiang Z, Wan W, Yang D. Sequencing and characterization of leaf transcriptomes of six diploid Nicotiana species. J Biol Res. 2016; 23(1):1-12.
65. Chopra R, Burow G, Farmer A, Mudge J, Simpson CE, Burow MD. Comparisons of De Novo Transcriptome Assemblers in Diploid and Polyploid Species Using Peanut (Arachis spp.) RNA-Seq Data. Plos One. 2014;9(12):e115055.

66. Hoang NV, Furtado A, Mason PJ, Marquardt A, Kasirajan L, Thirugnanasambandam PP, Botha FC, Henry RJ. A survey of the complex transcriptome from the highly polyploid sugarcane genome using fulllength isoform sequencing and de novo assembly from short read sequencing. BMC Genomics. 2017;18(1):395.

67. Ferreira de Carvalho J, Poulain J, Da Silva C, Wincker P, Michon-Coudouel S, Dheilly A, Naquin D, Boutte J, Salmon A, Ainouche M. Transcriptome de novo assembly from next-generation sequencing and comparative analyses in the hexaploid salt marsh species Spartina maritima and Spartina alterniflora (Poaceae). Heredity. 2013;110(2):181-93.

68. Gutierrez-Gonzalez JJ, Zheng JT, Garvin DF. Analysis and annotation of the hexaploid oat seed transcriptome. BMC Genomics. 2013;14(1):471.

69. Nakasugi K, Crowhurst R, Bally J, Waterhouse P. Combining transcriptome assemblies from multiple de novo assemblers in the Allo-tetraploid plant Nicotiana benthamiana. PLoS One. 2014;9(3):e91776.

70. Adams KL, Wendel JF. Polyploidy and genome evolution in plants. Curr Opin Plant Biol. 2005;8(2):135-41.

71. Cui L, Wall PK, Leebensmack JH, Lindsay BG, Soltis DE, Doyle JJ, Soltis PS, Carlson JE, Arumuganathan K, Barakat A. Widespread genome duplications throughout the history of flowering plants. Genome Res. 2006;16(6):738.

72. Zhang R, Calixto CP, Marquez Y, Venhuizen P, Tzioutziou NA, Guo W, Spensley M, Entizne JC, Lewandowska D, Ten HS. A high quality Arabidopsis transcriptome for accurate transcript-level analysis of alternative splicing Nucleic Acids Res. 2017:45(9):5061-73.

73. Conesa A, Madrigal P, Tarazona S, Gomezcabrero D, Cervera A, Mcpherson A, Szcześniak MW, Gaffney DJ, Elo LL, Zhang X. A survey of best practices for RNA-seq data analysis. Genome Biol. 2016;17(1):13.

74. Nierman WC, Arnab P, Anderson MJ, Wortman JR, Kim HS, Javier A, Matthew B, Keietsu A, Archer DB, Clara B. Genomic sequence of the pathogenic and allergenic filamentous fungus Aspergillus fumigatus. Nature. 2005:438(7071):1151

75. Galagan JE, Calvo SE, Borkovich KA, Selker EU, Read ND, David J, William FH, Li-Jun M, Serge S, Seth P. The genome sequence of the filamentous fungus Neurospora crassa. Nature. 2003.

76. Bolger AM, Lohse M, Usadel B. Trimmomatic: a flexible trimmer for Illumina sequence data. Bioinformatics. 2014;30(15):2114-20.

77. Xia EH, Li FD, Tong W, Li PH, Wu Q, Zhao HJ, Ge RH, Li RP, Li YY, Zhang ZZ, et al. Tea plant information archive: a comprehensive genomics and bioinformatics platform for tea plant. Plant Biotechnol J. 2019;17(10):19381953

78. Kent WJ. BLAT-the BLAST-like alignment tool. Genome Res. 2002;12(4): 656-64.

79. Chen C, Xia R, Chen H, He Y. TBtools, a toolkit for biologists integrating various biological data handling tools with a user-friendly interface. BioRxiv. 2018;289660.

80. Simão FA, Waterhouse RM, loannidis P, Kriventseva EV, Zdobnov EM. BUSCO: assessing genome assembly and annotation completeness with single-copy orthologs. Bioinformatics. 2015;31(19):3210-2.

81. Langmead B, Salzberg SL. Fast gapped-read alignment with bowtie 2. Nat Methods. 2012;9(4):357.

82. Dewey CN, Li B. RSEM: accurate transcript quantification from RNA-Seq data with or without a reference genome. Bmc Bioinformatics. 2011;12(1):323.

\section{Publisher's Note}

Springer Nature remains neutral with regard to jurisdictional claims in published maps and institutional affiliations. 